\title{
Reducing passengers' travel time by optimising stopping patterns in a large-scale network: A case-study in the Copenhagen Region
}

\author{
Parbo, Jens; Nielsen, Otto Anker; Prato, Carlo Giacomo
}

Published in:

Transportation Research. Part A: Policy \& Practice

Link to article, DOI:

10.1016/j.tra.2018.04.012

Publication date:

2018

Document Version

Peer reviewed version

Link back to DTU Orbit

Citation (APA):

Parbo, J., Nielsen, O. A., \& Prato, C. G. (2018). Reducing passengers' travel time by optimising stopping patterns in a large-scale network: A case-study in the Copenhagen Region. Transportation Research. Part A: Policy \& Practice, 113, 197-212. https://doi.org/10.1016/j.tra.2018.04.012

\section{General rights}

Copyright and moral rights for the publications made accessible in the public portal are retained by the authors and/or other copyright owners and it is a condition of accessing publications that users recognise and abide by the legal requirements associated with these rights.

- Users may download and print one copy of any publication from the public portal for the purpose of private study or research.

- You may not further distribute the material or use it for any profit-making activity or commercial gain

- You may freely distribute the URL identifying the publication in the public portal 
Reducing passengers' travel time by optimising stopping patterns in a large-scale network: A case-study in the Copenhagen Region

Jens Parbo a , Otto A. Nielsen ${ }^{\text {a }}$, Carlo G. Prato ${ }^{\text {b* }}$

a Department of Management Engineering, Technical University of Denmark

Bygningstorvet 116B, 2800 Kgs. Lyngby, Denmark

${ }^{\mathrm{b}}$ School of Civil Engineering, The University of Queensland

St Lucia 4072, Brisbane, Australia

* corresponding author

School of Civil Engineering, The University of Queensland

St Lucia 4072, Brisbane, Australia

email: c.prato@uq.edu.au 


\begin{abstract}
Optimising stopping patterns in railway schedules is a cost-effective way to reduce passengers' generalised travel costs without increasing train operators' costs. The challenge consists in striking a balance between an increase in waiting time for passengers at skipped stations and a decrease in travel time for through-going passengers, with possible consequent changes in the passenger demand and route choices. This study presents the formulation of the skip-stop problem as a bi-level optimisation problem where the lower level is a schedulebased transit assignment model that delivers passengers' route choices to the skip-stop optimisation model at the upper level, and where the upper level in return provides an improved timetable to the lower level. A heuristic method for large-scale urban networks is presented to solve this extremely complex bi-level problem, where the skip-stop optimisation is a mixed-integer problem, whereas the route choice model is a non-linear non-continuous mapping of the timetable. The method was tested on the suburban railway network in the Greater Copenhagen Region (Denmark): the reduction in railway passengers' in-vehicle travel time was $5.5 \%$, the reduction in passengers' generalised travel cost was $3.2 \%$ and, at the system level, the yearly consumer surplus amounted at 76.7 million DKK (about 10.3 million EUR or 12.7 million USD) when compared to the existing stopping patterns.
\end{abstract}

Keywords: public transport passengers' behaviour; stopping patterns; public transport optimisation; railway timetabling; large-scale networks. 


\section{Introduction}

Attempts to increase public transport ridership by reducing travel times and increasing reliability are often pursued via very costly investments in rolling stock and new or improved infrastructure. However, similar benefits may be obtained also via alternative cost-effective ways that involve the modification of line planning and stopping patterns (see, e.g., Leiva et al., 2010; Tetreault and El-Geneidy, 2010; Fu et al., 2016). This study focuses on optimising stopping patterns in railway networks with the aim of minimising passengers' travel cost while reducing the risk of delay.

When changing stopping patterns and updating timetables, planners have to compromise between the desires of the passengers, who want to receive a service with high frequency and high speed, and the wishes of the operators, who want to maximise their profit by (for example) minimising fleet and crew sizes (Ceder, 2015). This study presents a method that aims at being beneficial to both passengers and operators by minimising the generalised travel cost of the former as well as the cost of the latter. Specifically, the method updates timetables by proposing stopping patterns configurations based on a socioeconomic objective function that considers both passengers' consumer surplus and operators' costs. Moreover, the method takes into account the possibility that passengers might adapt their travel behaviour given the update of the timetables with the changed stopping patterns. To this extent, a bi-level problem is proposed to solve iteratively a skip-stop optimisation problem and a scheduled-based transit assignment that allows to optimise stopping patterns and to account for adapting travel behaviour.

The contribution of this study is twofold. Firstly, existing skip-stop methods assume that the route choices of passengers are not affected by the changes in the stopping patterns. The present study overcomes this limitation by representing the adaptation in travel behaviour of the passengers after the timetables have been modified. Secondly, existing skip-stop methods analyse simple networks (generally composed of one corridor). The present study proposes a method that is then applied to a large-scale suburban network at the traveller level, specifically the railway network of the Greater Copenhagen Area where passenger loads are considered for individual trains in each track segment.

The remainder of the paper reviews existing literature in section 2, formulates the problem in section 3 , presents a solution algorithm in section 3 , and shows results for the suburban network in section 5. Conclusions and avenues for further research are then illustrated in section 6 . 


\section{Literature review}

The literature about the modification of stopping patterns has origin in the pioneering work of Kikuchi and Vuchic (1982), who examined the effect of skipping stops on the access time, waiting time and in-vehicle travel time of passengers. Specifically, their findings revealed that access and waiting times increased for waiting passengers because of the decreased frequency of the service after modifying the stopping patterns. Moreover, invehicle travel time decreased for through-going passengers because of the reduced times for deceleration, dwell and acceleration. This review focuses initially on existing skip-stop optimisation methods from two perspectives, namely the consideration of passenger behaviour and the formulation of solution algorithms. Then, the review considers the importance of accounting for changes in passenger route choices and concentrates on railway operation studies where changes in passenger behaviour are taken into account.

\subsection{Skip-stop optimisation}

Skip-stop optimisation in transit networks has attracted an increasing amount of attention in recent years. Existing methods aimed at minimising either passengers' travel time (e.g., Suh et al., 2002; Mesa et al., 2009; Jong et al., 2012; Sogin et al., 2012; Feng et al., 2013; Jamili et al., 2014; Katori et al., 2014; Lee et al., 2014) or a combination of passengers' travel time and operating costs (e.g., Leiva et al., 2010; Freyss et al., 2013; Lin and Ku, 2014; Chen et al., 2015). Existing methods were formulated typically as mixed integer linear problems that were computationally hard to solve for real-world networks, were tested usually on a single railway corridor for the purpose of reducing the complexity of solution algorithms, and did not include adaptation of passenger behaviour as a consequence of the changes in the timetables related to the modifications in the stopping patterns.

Differences could be observed across studies in the formulation of the problem and the proposal of solution algorithm. Suh et al. (2002) and Mesa et al. (2009) proposed to skip smaller stops in the network. Leiva et al. (2010) designed an optimisation method that minimised user costs by first separating the lines between all-stop services, express services (i.e., origin and destination stops were served), and skip-stop services (i.e., a subset of stops were served), and then solving analytically the problem to find a local minimum before applying a heuristic to prove the fitness of the solution. Freyss et al. (2013) applied a continuous approximation method to find the optimal distance between visited stops and, implicitly, also the best performing stopping patterns. Jamili et al. (2014) applied a fuzzy approach to reach a flexible solution with the aim of minimising travel time and maximising the train spread while relaxing the headway constraints. Katori et al. (2014) applied dynamic 
programming to find optimal stopping patterns and potential overtaking stops after forming local timetables from train diagrams.

Table 1 presents existing skip-stop optimisation methods according to two perspectives: the first perspective looks at the consideration of passenger behaviour, while the second perspective looks at the approach of solution algorithms. While the first perspective separates the studies with predetermined static demand from the ones with approximated static demand, the second perspectives differentiates between heuristic and dynamic programming approaches.

Static demand was used in the existing methods either as predetermined, often based on passenger counts or ticket sales (e.g., Suh et al., 2002; Mesa et al., 2009; Leiva et al., 2010; Jong et al., 2012; Feng et al., 2013; Jamili et al., 2014; Katori et al., 2014; Lee et al., 2014; Lin and $\mathrm{Ku}, 2014$ ), or approximated, generally because of complete or partial data inavailability (Sogin et al., 2012; Freyss et al., 2013; Chen et al., 2015). Specifically, Freyss et al. (2013) did not have data about passenger movements and hence assumed that the destinations of all boarding passengers were evenly distributed on all remaining stops, while Sogin et al. (2012) and Chen et al. (2015) had data only about travel time between stops and hence ignored waiting and transfer times. Obviously, these approximations are not ideal as the calculation of the benefit for skipping a certain stop requires knowing whether the onboard passengers' surplus (i.e., the reduced travel time) exceeds the boarding and alighting passengers' cost (i.e., the increased waiting time). Not having information makes this calculation impossible at the stop level, while having only information on travel time makes this calculation inaccurate when considering that waiting and transfer times are more costly than in-vehicle times (e.g., Raveau et al., 2014; Anderson et al., 2017) and lead to inaccurate passenger flows (Nielsen, 2000). However, predetermined demand is also not ideal as it is conceivable that the changes in in-vehicle times, waiting times and transfer times affect the behaviour of passengers that might search for alternative routes. The present study addresses the gap in the skip-stop optimisation literature by considering passenger behaviour as responsive to the updated timetables.

Dynamic programming was used to simple networks with one corridor as solving the skip-stop problem for real-world networks is not feasible within reasonable calculation time in practice (Lin and $\mathrm{Ku}, 2014$ ). Heuristic approaches are able to find solutions fast in larger problems and the present study proposes a solution algorithm that uses a heuristic method. 
Table 1. Overview of skip-stop optimisation approaches

Passenger demand

\begin{tabular}{|c|c|c|c|c|}
\hline & & \multicolumn{3}{|c|}{ Passenger demand } \\
\hline & & \multicolumn{2}{|c|}{ Static } & \multirow[t]{2}{*}{ Responsive } \\
\hline & & Predetermined & Approximated & \\
\hline \multirow{2}{*}{$\begin{array}{l}\text { Solution } \\
\text { Method }\end{array}$} & Heuristic & $\begin{array}{c}\text { Leiva et al. (2010) } \\
\text { Jong et al. (2012) } \\
\text { Feng et al. (2013) } \\
\text { Lin \& Ku (2014) } \\
\text { Lee et al. (2014) }\end{array}$ & $\begin{array}{l}\text { Sogin et al. (2012) } \\
\text { Freyss et al. (2013) } \\
\text { Chen et al. (2015) }\end{array}$ & Current study \\
\hline & $\begin{array}{l}\text { Dynamic } \\
\text { Programming/ } \\
\text { Other }\end{array}$ & $\begin{array}{l}\text { Suh et al. (2002) } \\
\text { Mesa et al. (2009) } \\
\text { Jamili et al. (2014) } \\
\text { Katori et al. (2014) }\end{array}$ & - & - \\
\hline
\end{tabular}

\subsection{Responsive passenger behaviour to railway operation changes}

Given the individuated gap in the skip-stop optimisation literature, this part of the review looks at studies focusing on responsive passenger behaviour to railway operation changes, and more specifically to explicit adaptation of passenger route choices as a consequence of changes in railway operations (Sun et al., 2014).

The most typical approach consists in embedding a traffic assignment model within the optimisation model. Constantin and Florian (1995) formulated a bi-level frequency optimisation problem that minimised expected travel and waiting times by changing frequency settings at the upper level, and adapting route choices according to a traffic assignment model at the lower level. Wang and Lin (2010) formulated a bi-level cost minimisation problem that minimised transit operating costs related to fleet size by changing headways settings at the upper level, and updating route choices according to a traffic assignment model at the lower level. Parbo et al. (2014) formulated a bi-level time minimisation problem that minimised passengers' waiting times by optimising departure times for buses at the upper level, and recalculating route choices according to a traffic assignment model at the lower level.

Similarly, line planning problems have considered responsive demand. Schöbel and Scholl (2006) formulated a line planning problem that minimised in-vehicle times and operating costs obtained from a frequency-based assignment model. However, Borndörfer et al. (2007) pointed out that the problem required a reduction in complexity by minimising only in-vehicle times, and hence unrealistically not penalising transfers, and by considering an application to a medium-size network. Bull et al. (2015) proposed a line planning problem that considered capacity constraints and passenger flows, although with even loads resulting from a frequency-based model. Rezanova et al. (2015) formulated a line planning problem 
that considered passenger inconvenience as derived from the line plan, frequency of each line, and capacity of the rolling stock assigned to each line. However, the problem disregarded travel time and number of transfers.

The present study took inspiration from the bi-level optimisation problems by proposing an upper level solving the skip-stop optimisation problem, and a lower level using an assignment model to calculate the changes in route choice behaviour because of the updated stopping patterns. Moreover, the present study formulated a schedule-based assignment model because of the limitations in the line planning studies, namely the fact that transfers were discarded (Borndörfer et al., 2007), loads on specific lines were not considered (Bull et al., 2015), and travel time and number of transfers were disregarded (Rezanova, 2015).

\section{Skip-stop and assignment bi-level problem formulation}

\subsection{Upper level problem: skip-stop optimisation}

Given a railway line with $n$ stops where $n-2$ stops are candidates to skip-stop optimisation (initial and terminal stops are not suitable candidates), the number $P$ of different stopping patterns is equal to $2^{n-2}$. Given a corridor where $L$ railway lines are operated, the number $P$ of different stopping patterns for each railway line is equal to the following binomial coefficient.

$$
\left(\begin{array}{l}
P \\
L
\end{array}\right)=\frac{P !}{L !(P-L) !}
$$

For example, given $L=2$ railway lines that operate on a corridor with $n=4$ stops, each railway line can have $P=4$ different stopping patterns, while the corridor can be operated in 16 different ways. Applying eq. (1), 6 different stopping patterns are possible and they can be justified according to the following considerations: of the 16 possible combinations, 4 can be disregarded as the 2 railway lines operate with similar stopping patterns, and 6 can be disregarded when considering mirroring operations (i.e., line 1 operates with pattern $\mathrm{A}$ and line 2 operates with pattern B mirrors when line 1 operates with pattern B and line 1 operates with pattern A).

The optimisation problem is written as follows:

$$
\min \left(\omega_{1} \sum_{i, j, c, r} C_{i j c r} \cdot T_{i j c r}+\omega_{2} \sum_{s} \sum_{k} \frac{1}{H_{\mathrm{s}, k, k+1}}\right)
$$

subject to:

$$
\begin{gathered}
H_{s, k, k+1}=\left(\left(b^{k+1, p}+t_{s}^{k+1, p}\right)-\left(b^{k, p}+t_{s}^{k, p}\right)\right) \cdot x_{l}^{p} \quad \forall l, k, s, p \\
H_{s, k, k+1} \geq H \quad \forall s, k
\end{gathered}
$$




$$
\begin{gathered}
C_{i j c r} \geq C_{i j c r}^{p}+M \cdot\left(1-a_{i j}^{p} \cdot x_{l}^{p}\right) \quad \forall i, j, r, c, l, p \\
\sum_{p} x_{l}^{p}=1 \quad \forall l \\
x_{l}^{p} \in\{0,1\} \quad \forall p, l \\
t_{s}^{k, p} \geq 0 \quad \forall k, p, s \\
b^{k, p} \geq 0 \quad \forall k, p \\
C_{i j c r} \geq 0 \quad \forall i, j, c, r \\
C_{i j c r}^{p} \geq 0 \quad \forall p, i, j, c, r
\end{gathered}
$$

where $i$ is the origin stop, $j$ is the destination stop, $s$ is a generic stop, $p$ is a stopping pattern $(p=1, \ldots, P), l$ is a railway line $(l=1, \ldots, L), r$ is a route, $k$ is a train run, and $c$ is a trip purpose. Moreover, $a^{p}{ }_{i j}$ is a parameter equal to 1 if $p$ visits both $i$ and $j$ (and 0 otherwise), $x^{p} l_{l}$ is a variable equal to 1 if railway line $l$ is operated with stopping pattern $p$ (and 0 otherwise), $b^{k, p}$ is the departure time for train run $k$ from the initial stop with stopping pattern $p, H$ is a minimum safety headway, and $M$ is a large positive constant. Accordingly, $H_{s, k, k+1}$ is the actual headway on stop $s$ between subsequently running train runs $k$ and $k+1, t^{k, p}$ is the travel time from the initial stop $i$ to stop $s$ for train run $k$ when stopping pattern $p$ is operated, $T_{i j c r}$ is the passenger demand on route $r$ between stops $i$ and $j$ for trip purpose $c, C^{p}{ }_{i j c r}$ is the generalised travel cost along route $r$ between stops $i$ and $j$ for trip purpose $c$ when stopping pattern $p$ is operated, and $C_{i j c r}$ is the minimum generalised cost of route $r$ between stops $i$ and $j$ for trip purpose $c$ among all operated stopping patterns. Lastly, $\omega_{1}$ and $\omega_{2}$ are weights for the two terms in the objective function.

The objective function (2) minimises a weighted sum of passengers' generalised cost and train operation heterogeneity. Heterogeneity refers to differences between trains in terms of speed, headways and stopping patterns: reducing heterogeneity with the same frequency of operations is equivalent to reducing capacity consumption, or in other words fewer trains can be run under heterogeneous operations within the same period when compared to homogeneous operations. Heterogeneity is measured as the Sum of Shortest Headway Reciprocals (SSHR, Vromans et al., 2006) to provide an indication of the change in capacity utilisation and the amount of buffer time between trains when comparing the existing and the optimised stopping patterns. The weights $\omega_{1}$ and $\omega_{2}$ of the objective function (2) give more or less emphasis on either component and enable to achieve consistency in the units of the two components. Constraint (3) expresses the headway between consecutive train runs $k$ and $k+1$ on every stop $s$ for each pattern $p$, while constraint (4) ensures that the headways are not 
below the minimum safety headway $H$. Notably, the objective function (2) and the headway constraints (3) and (4) are written to take into account explicitly the heterogeneity of the train operations in the objective function. Constraint (5) expresses the minimum generalised cost between stops $i$ and $j$ over the stopping pattern $p$ : if $i$ and $j$ are not visited in the stopping pattern $p$, this is set to a large number to ensure that all stops are visited, otherwise the objective will be penalised by $M$ multiplied by the demand $T_{i j c r}$. Constraint (6) ensures that each line is operated with one and only one stopping patterns. Last, constraints (7) through (11) are domain setting constraints.

\subsection{Lower level problem: transit assignment}

The lower level introduces the responses of the passengers to the changes in the timetables after the skip-stop optimisation at the upper level. A route choice model at a high level of detail in both time and space is used to capture the adaptation of the route choices of the passengers to the timetables.

The model is schedule-based and utility-based in order to analyse demand variations between different runs belonging to the same line as well as between different lines running on the same track segments, which in turn allows analysing actual changes in route choice behaviour following the changes in the stopping patterns. Moreover, the schedule-based model allows not only to derive the individual train run loads, but also avoiding the common lines problem that is typical of frequency-based models and affected previous studies working on responsive demand to train operation optimisation. The scheduled-based and utility-based approach is presented graphically in figure 1, and details about its formulation can be found in Nielsen (2004) and Nielsen et al. (2006).

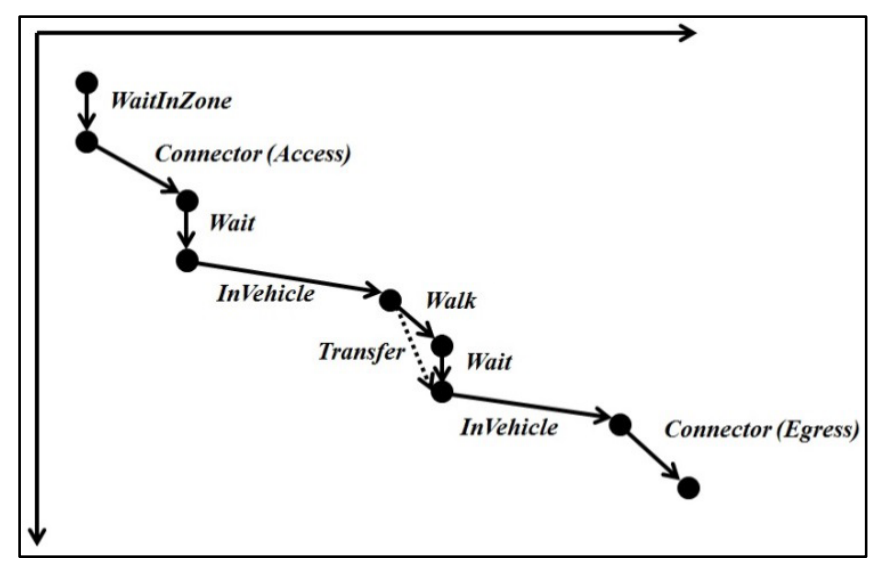

Figure 1. Transit assignment procedure

From the schedule-based perspective, each route $r$ is uniquely defined in a disaggregate time-space graph that allows calculating the passenger load for each train run $k$ over the route 
$r$. From the utility-based perspective, the generalised travel cost for passengers travelling on route $r$ from stop $i$ to stop $j$ for trip purpose $c$ is also uniquely defined:

$$
C_{i j c r}=\beta_{h t c} \cdot h t_{i j c r}+\beta_{a t c} \cdot a t_{i j c r}+\beta_{t t c} \cdot w a t_{i j c r}+\beta_{t w t c} \cdot t w t_{i j c r}+\beta_{n t c} \cdot n t_{i j c r}+\beta_{i v t, c} \cdot i v t_{i j c r}
$$

where, for route $r$ between stops $i$ and $j$ for trip purpose $c, C_{i j c r}$ is the minimum generalised cost, $h t_{i j c r}$ is the waiting time at home or in the origin zone, $a t_{i j c r}$ is the access time from home or the origin zone to the desired boarding stop, wat ${ }_{i j c r}$ is the walking time to the desired boarding stop, $t w t_{i j c r}$ is the walking transfer time for the transfers on the route, $n t_{i j c r}$ is the number of transfers on the route, and $i v t_{i j c r}$ is the in-vehicle time. The respective parameters are calibrated to reflect the travel behaviour by matching the passenger counts, and they reflect the sensitivity of the passenger to the components of the generalised travel cost associated with a journey via route $r$ between stops $i$ and $j$ for trip purpose $c$.

The lower-level problem is solved by minimising the generalised travel cost to obtain passenger loads on each run that result in travel times $T_{i j c r}$ between stations $i$ and $j$ based on the stopping patterns $p$. It should be noted that we do not constrain the problem to any specific schedule-based assignment model, as any solution (see, e.g., Ceder, 2015; Gentile and Noekel, 2016) would serve the purpose. The schedule-based in the specific application of this study is formulated according to Nielsen et al. (2006) with the modifications presented in Anderson et al. (2016).

The demand and the travel times obtained at the lower level are the response to the mapping of the schedule optimisation at the upper level, while in turn the skip-stop optimisation at the upper level receives the updated demand and travel times as input.

\section{Bi-level problem solution}

The bi-level problem is intended for solving large-scale networks that introduce a high degree of complexity (e.g., Sogin et al., 2012; Jong et al., 2012). Accordingly, a bi-level heuristic solution method solving the two problems sequentially is developed: the skip-stop optimisation is solved via a heuristic algorithm and is then integrated with the schedule-based assignment model.

\subsection{Requisites and assumptions}

Two requisites exist for the solution of the bi-level problem formulated in section 3 . The first requisite consists in the availability of a railway network and a timetable that allow detailing when each train arrives at and departs from each stop $s$. The second requisite is that the schedule-based transit assignments details the passengers on each train, and hence also the number of boarding, alighting and through-going passengers at each stop $s$. 
Three assumptions are made for solving the bi-level optimisation problem. The first assumption concerns the configuration of the corridors, namely railway lines on a corridor are subject to skip-stop optimisation when they satisfy the following conditions:

- At least two railway lines run on the same tracks.

- Railway lines in the corridor run at a high frequency.

- The capacity usage in the corridor is below maximum.

The first condition ensures that all stops are served by at least one railway line, otherwise the only railway line would follow an all-stop pattern. It should be noted that, when changing stopping patterns for the railway lines operating in the corridor, the number of railway lines operating with different stopping patterns has to be the same before and after the optimisation so that the structure is maintained and the passengers' confusion following the timetable update is reduced. The second condition ensures that skip-stop services are implemented successfully in that the passengers do not experience excessively high waiting times (Freyss et al., 2013). The third condition ensures that the minimum safety headway constraints are not violated when changing stopping patterns, since skipping stops might force trains to slow down when overtaking is not possible (e.g., because of the track layout, non-existing side tracks) due to trains ahead when capacity utilisation is close to maximum.

The second assumption concerns the stops to be considered:

- Skipping transfer stops is prohibited.

- Skipping initial and terminal stops is prohibited.

The first condition avoids closing transfer stops that could have a severe impact on the passengers' travel experience since they would be forced to find alternative routes that are significantly different from the chosen ones. The second condition ensures that the line structure is conserved.

The third assumption concerns overtaking since at some point a train, after skipping several stops, might have to slow down and wait for the train ahead with a consequent loss of the travel time savings from skipping stops. This study represents overtaking by considering the stops in the corridor sequentially from one end to the other and limiting the difference in the number of skipped stops between parallel railway lines (similar to Lee et al., 2014). The headway is thereby kept larger than the sum of the safety distance and the travel time savings obtained from skipping stops, thus ensuring that the minimum safety headway constrains are not violated and no trains are forced to wait behind or overtake other trains. 


\subsection{Heuristic solution method}

The bi-level problem is solved sequentially via a heuristic solution method where the output of the upper (lower) level serves as the input to the lower (upper) level until convergence is reached. The heuristic solution method follows the step-wise method elaborated in the following sub-sections.

0. Initialise all railway lines to all-stop lines and run the transit assignment. Create a list $F$ of all the stops s subject to skip-stop optimisation.

1. Derive the pre-optimisation potential for each stop $s$ in $F$.

2. Derive the elaborate optimisation potential for the most promising stop ${ }^{*}$ in F.

3. If $s^{*}$ has a positive optimisation potential, skip stop $s^{*}$ and remove the sub-network of $s^{*}$ from $F$. Otherwise, remove only stop $s^{*}$ from $F$ and return to step 2.

4. If $F$ is not empty, return to step 2. Otherwise, continue.

5. Stop if the stopping criterion is met.

Otherwise, run the transit assignment and return to step 1.

In the initialisation step 0 , the first lower level calculation consists in initialising all railway lines to all-stop lines and running the schedule-based assignment model. The rationale behind this step is to avoid that existing stopping patterns (based on old demand patterns) bias the new stopping patterns (based on the adapted demand patterns). Steps 1 and 2 derive the potential for skipping the most promising stop $s^{*}$ on a particular railway line. Step 3 performs the skip-stop optimisation and removes all stops related to $s^{*}$ from the list $F$ of all stops that could be skipped. Step 4 continues the upper level calculations by skipping stops until list $F$ is empty. Step 5 performs a new lower level calculation, namely runs a traffic assignment with the new stopping patterns, unless the stopping criterion is met.

\subsubsection{Optimisation potential}

In step 1, a pre-optimisation potential is calculated for every stop $s$ to select the most promising stop $s^{*}$ and then calculate an elaborate optimisation potential. The data requirements for calculating the optimisation potential are presented in figure 2: in line with the assumptions, all railway lines and their timetables are known as well as the sum of embarking and disembarking passengers. 


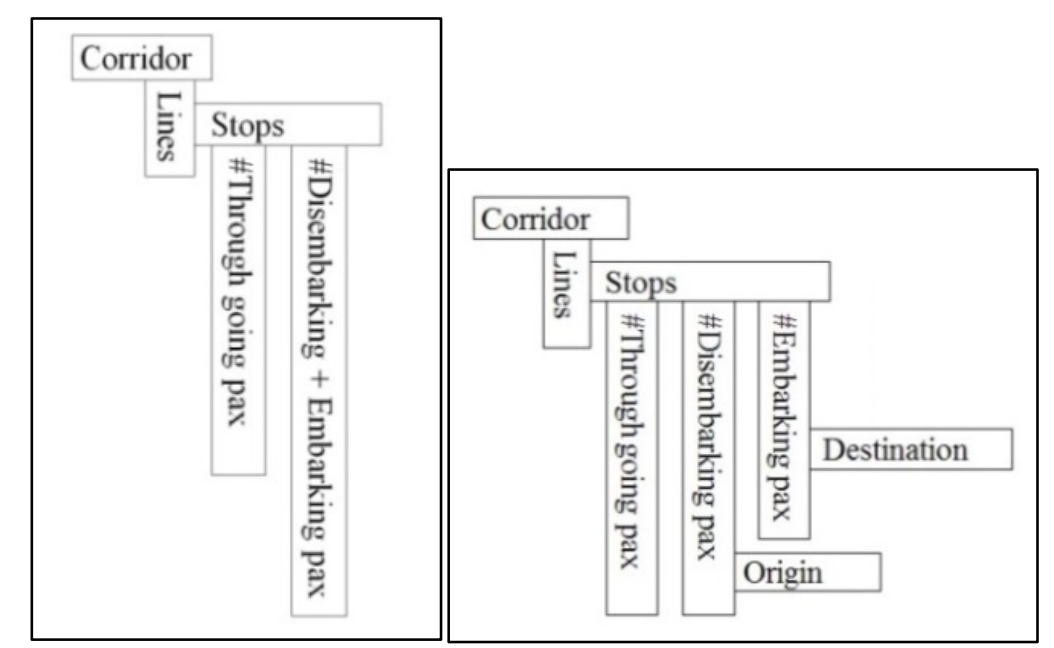

Figure 2. Calculation graphs outlining the data requirements for preliminary and elaborate optimisation potential

Given the data, the decision about whether or not to consider a certain stop on a particular railway line as a preliminary skip-stop candidate relies on the following calculation:

$$
P O P_{f l s}=\frac{T_{f l s}}{T D_{f l s}+T E_{f l s}} \quad \forall f, l, s
$$

where $P O P_{f l s}$ is the pre-optimisation potential for each stop $s$ on railway line $l$ running in corridor $f, T_{f l s}$ is the number of through-going passengers at stop $s$ on line $l$ in corridor $f$, and $T D_{f l s}$ and $T E_{f l s}$ are respectively the numbers of passengers disembarking and embarking at stop $s$ on line $l$ in corridor $f$.

Following the identification of the most promising stops on the basis of their preoptimisation potential $P O P_{f l s}$, an elaborate optimisation potential is calculated for a comprehensive assessment of the benefits of skipping a certain stop $s$. As represented in figure 2, the elaborate optimisation potential requires disaggregate data on passengers' route choices to derive the effects of the changes in the timetables on the components of the generalised travel cost.

\subsubsection{Elaborate optimisation potential (with adapted route choices)}

In step 2, the additional piece of information considered for the calculation of the extended optimisation potential concerns the origin stations of the disembarking passengers and the destination stations of the embarking passengers (given railway lines, timetables, and disembarking and embarking passengers at each stop). 
Four types of trip adaptations are available on the basis of the way that passengers are affected when their desired boarding/alighting stop is skipped, both within (trip types 1, 2, 3) and outside (trip type 4) the corridor. The four types are explained in detail in the following paragraphs for trips destined to a stop that is a skip-stop candidate, while (given the obvious similarity) they are omitted for trips originated from a stop that is a skip-stop candidate. The example in figure 3, where the skip-stop candidate is stop 4 on railway line B, provides a graphic support to the explanations.

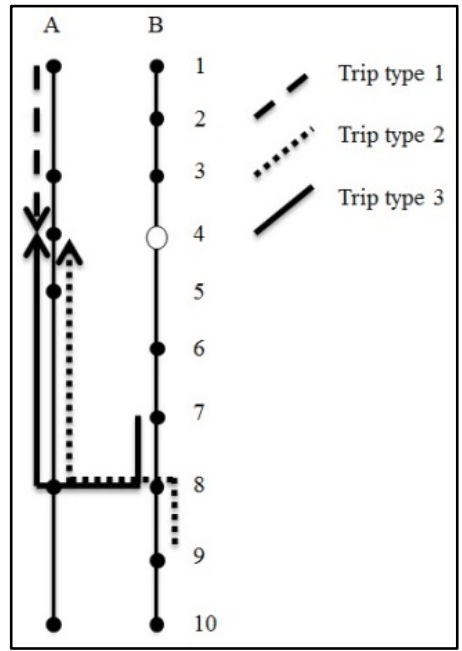

Figure 3. Graphic representation of the trip type adaptations

The three possible types of trip adaptation within the corridor are as follows:

1. At least one other railway line in the corridor serves the affected passengers' boarding and alighting stops.

2. Boarding and alighting stops are not served by any railway line in the corridor, but there exists a transfer stop in-between where two railway lines serving respectively the boarding and alighting stops meet.

3. Boarding and alighting stops are not served by any railway line in the corridor and there are no transfer possibilities in-between, thus at some point the affected passengers have to travel in the opposite direction.

In trip type 1, passengers are assumed to arrive randomly at the boarding stop because of the high frequency of the railway lines operated in the corridor. Depending on their arrival time at the boarding stop, they might be unable to board the first departing train if it does not serve both their boarding and alighting stops, and they might experience an additional cost because of the extra waiting time. The additional cost $C 1_{i j l c}$ is equal to the increase in waiting time (assumed equal to half the additional headway between $h n e w_{i j l}$ with the new pattern and $h_{i j l}$ with the old pattern) for railway lines $l$ serving both stops $i$ and $j$, multiplied by the 
number of passengers $T_{i j l c}$ travelling between stops $i$ and $j$ and having value of time of waiting $\beta_{h t, c}$ for their trip purpose $c$.

$$
C 1_{i j l c}=\frac{1}{2}\left(\text { hnew }_{i j l}-h_{i j l}\right)\left(\beta_{h t, c} \cdot T_{i j l c}\right) \quad \forall i, j, l, c
$$

In trip type 2, passengers might still experience additional cost because they might be unable to board the first departing train. Moreover, passengers experience additional generalised travel cost because of the transfer at a stop that serves two railway lines, where one also serves the boarding stop and the other serves the skip-stop candidate. The additional $\operatorname{cost} C 2_{i j l c}$ is equal to the sum of the waiting time at the boarding stop, the transfer time conservatively set equal to the headway $h_{j s * l}$ of railway lines $l$ serving the transfer stop $j$ and the skip-stop candidate $s^{*}$, and a transfer penalty $t p_{c}$, multiplied by the number of passengers $T_{i j l c}$ travelling between stops $i$ and $j$ and having value of time $\beta_{h t, c}$ and transfer penalty $\beta_{n t, c}$ for their trip purpose $c$.

$$
C 2_{i j l c}=\left(\beta_{h t, c} \cdot\left(\frac{1}{2} h_{i j l}+h_{j s^{*} l}\right)+\beta_{n t, c} \cdot t p_{c}\right) \cdot T_{i j l c} \quad \forall i, j, l, c
$$

In trip type 3, passengers might still experience additional cost because they might be unable to board the first departing train and they have a transfer. Moreover, completing the trip requires travelling in the opposite direction and an extra transfer, which might discourage passengers from choosing the path due to reluctance to travel on routes diverging significantly from the direct one (Raveau et al., 2014). Accordingly, skipping the stop is heavily penalised and the additional cost $C 3_{i j l c}$ is equal to $M$ (a large number) multiplied by the number of passengers affected, implying that these trips are allowed only when a negligible amount of passengers travel between the stops $i$ and $j$.

$$
C 3_{i j l c}=M \cdot T_{i j l c} \quad \forall i, j, l, c
$$

In the fourth type of trip adaptation, passengers travelling from outside the corridor to the skip-stop candidate are assumed to be affected only by the reduced frequency of railway lines serving the skip-stop candidate. The reduced frequency is calculated as the difference between the old and the new headway, as passengers wait for a train serving both boarding and alighting stops rather than boarding the first arriving train and then making a transfer. The additional cost $C 4_{i j l c}$ is equal to the headway increase for railway lines $l$ serving stops $i$ and $j$, multiplied by the number of passengers $T_{i j l c}$ travelling between stops $i$ and $j$ and having value of time $\beta_{h t, c}$ for their trip purpose $c$.

$$
C 4_{i j l c}=\frac{1}{2}\left(\text { hnew }_{i j l}-h_{i j l}\right)\left(\beta_{h t, c} \cdot T_{i j l c}\right) \quad \forall i, j, l, c
$$


Considering each pair of stops $i$ and $j$, the total additional cost is calculated as:

$$
A d d C_{i j l c}=x_{1} C 1_{i j l c}+x_{2} C 2_{i j l c}+x_{3} C 3_{i j l c}+x_{4} C 4_{i j l c} \quad \forall i, j, l, c
$$

where the $x_{t}$ 's are equal to one if stops $i$ and $j$ are connected by a type $t$ trip, and zero otherwise. As acceleration, deceleration and dwell time are saved at the skipped stop, a total additional benefit is calculated as the reduction of in-vehicle travel time between IVTnewijl with the new stopping pattern and $I V T_{i j l}$ with the old one.

$$
\text { Benefit }_{i j}=\left(\text { IVTnew }_{i j l}-I V T_{i j l}\right) \cdot T_{i j l c} \cdot \beta_{i v t, c} \quad \forall l, i=s \vee j=s, c
$$

If the elaborate optimisation potential shows that the benefits exceeds the total additional costs, then the candidate stop is skipped.

\subsubsection{Skip-stop and sub-network removal}

In step 3, when the stop $s^{*}$ is skipped, the sub-network of $s^{*}$ is removed from the list $F$. Consider in fact that, if all the stops could be skipped at the same time in the upper level, the passenger flows from the previous iteration would deviate excessively and the algorithm would risk not to converge (or at least to oscillate and converge very slowly).

As a tool for reducing calculation time by limiting the number of lower level calculations without compromising the accuracy of the elaborate optimisation potential, sub-networks with minor passenger interactions are constructed for each stop. Specifically, sub-networks are created as stops are skipped and, given a certain skipped stop, it is not possible to skip other stops within that stop's sub-network. The sub-network of a stop consists of two parts:

- For the affected railway line, the remaining stops in the corridor.

- For the other railway lines in the corridor, the skipped stop.

Prohibiting stops from the sub-network to be skipped reduces the difficulty in predicting passengers' route choice changes when these stops are skipped. In fact, when skipping a stop leads to a large change in passenger shares between the railway lines operating in the corridor, it becomes more complex to predict the impact of skipping additional stops on the same railway line. Figure 4 presents a graphic representation of an example of sub-network formation as a compromise between being able to predict the passengers' adapted route choices and allowing as many stops as possible to be skipped before running the next traffic assignment. 


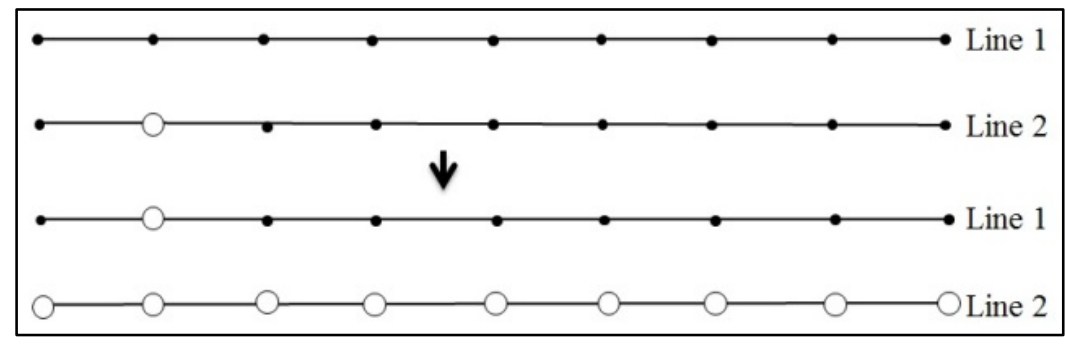

Figure 4. Sub-network formation in a two-line corridor

\subsubsection{Stopping criterion}

After step 4 verifies that the list $F$ of candidate stops is empty, repeating the last two steps in the case it is not, step 5 verifies that convergence is achieved. The stopping criterion states that the bi-level skip-stop optimisation and traffic assignment method is terminated when no stop has an elaborate optimisation potential where benefits exceed costs. Overall, the following pseudo-code elaborates the threads from the previous sub-sections:

\section{Pseudo-code}

The pseudo-code gathers the threads from the previous sub-sections.

Initialisation, Run transit assignment

\section{Upper-level problem}

Calculating optimisation potentials of skipping stops

For each corridor $f$

For each railway line $l$

For each stop $s$

Calculate optimisation potentials $P O P_{f l s}$

Store values $\left(f, l, s, P O P_{f l s}\right)$ in a list $F$

$$
\text { If } P O P_{f l s}<0 \text {, discard } s
$$

If $F$ is empty, terminate the entire process

Otherwise, proceed

\section{Skipping stops}

Continue the following until $F$ is empty

Select the stop $s^{*}$ with the largest $O P$ in $F$

Calculate the elaborate optimisation $E O$ potential for $s^{*}$

If $E O>0$, and no exceptions are violated, skip stop $s^{*}$

Otherwise, remove $s^{*}$ from $F$ and select new $s^{*}$

Derive sub-network $s n$ for $s^{*}$

Remove all values $s n$ from $F$

\section{Go to the lower-level problem}

\section{Lower-level roblem}

\section{Run the transit assignment}

Calculate the solution value and obtain costs and times

Go to the upper-level problem.

A couple of considerations apply. Firstly, the relevant information from the traffic assignment to be passed to the skip-stop optimisation is the number of passengers that are through, disembarking or embarking at each stop $s$ for each line $l$ and each train run $k$ over route $r$. Secondly, the described method strikes a balance between having greater emphasis on passengers' travel behaviour and investing in the optimisation. Thirdly, the described method has been thought for large-scale applications rather than traditionally used small test 
networks because of the need to provide solutions to metropolitan networks facing congestion problems and dealing with limited resources.

\section{Large-scale case-study}

The bi-level heuristics solution method was tested on the suburban railway network in the Greater Copenhagen Area in Denmark, as presented in figure 5. The network resembles similar European metropolitan urban rail systems that operate at distances and frequencies between high frequent metros and longer distance regional trains (e.g., Stockholm, Oslo, Berlin, Munich, Zurich, Paris). The network has 7 lines, 84 stations and 34 relevant transfer stations between skip-stop lines.

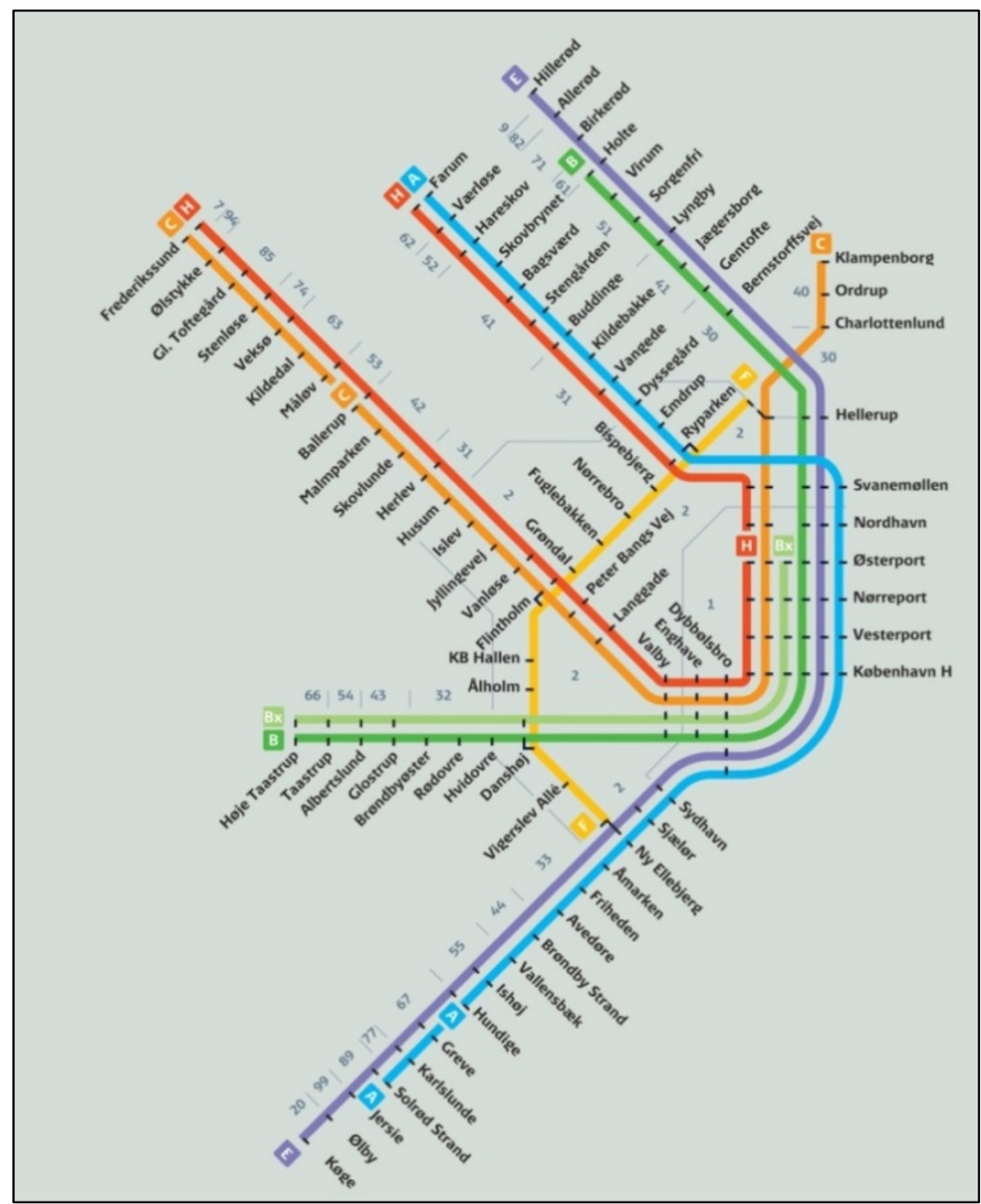

Figure 5. The suburban railway network of the Greater Copenhagen Area

The analysis focused on the morning peak hours (7am - 9am) where the system was measured by a counting train system (Nielsen et al., 2014) and passenger matrices were estimated by a Multipath Matrix Estimation Procedure, MPME (Nielsen, 1998). 
Five corridors where considered for testing the bi-level problem and solution: (i) Dybbølsbro - Køge, (ii) Danshøj - Høje Taastrup, (iii) Flintholm - Ballerup, (iv) Ryparken Farum, and (v) Hellerup - Holte. Each corridor has 2 railway lines and all corridor patterns can be combined. Some stations were not candidate according to the assumptions described in section 4, which implied that corridor (i) could skip up to 12 stops, (ii) up to 5, (iii) up to 12, (iv) up to 8 and (v) up to 5. This is a very large-scale problems that results in 16.8 million possible combinations, as described in table 2, which correspond to the combinatorial challenge of the network considered for the case-study. Considering that the schedule-based assignment model converges in about one hour of calculation time, a brute force evaluation of all combinations would take 16.8 million hours (about 1918 years).

Table 2. Possible number of different skip-stop patterns

\begin{tabular}{|l|rrrrr|r|}
\hline Line / skipped stops & $\begin{array}{l}\text { Dybbølsbro } \\
- \text { Køge }\end{array}$ & $\begin{array}{c}\text { Danshøj - } \\
\text { Høje } \\
\text { Taastrup }\end{array}$ & $\begin{array}{c}\text { Flintholm } \\
\text { - Ballerup }\end{array}$ & $\begin{array}{c}\text { Ryparken } \\
- \text { Farum }\end{array}$ & $\begin{array}{c}\text { Hellerup }- \\
\text { Holte }\end{array}$ & Total \\
\hline$n$ stops & 12 & 5 & 12 & 8 & 5 & \\
$L$ lines & 2 & 2 & 2 & 2 & 2 & \\
$P$ patterns $\left(\mathrm{P}=2^{\mathrm{n}-2}\right)$ & 4,096 & 32 & 4,096 & 256 & 32 & \\
\hline $\begin{array}{l}\text { Combinations per } \\
\text { corridor (eq. 1) }\end{array}$ & $8,386,560$ & 496 & $8,386,560$ & 32,640 & 496 & $16,806,752$ \\
\hline
\end{tabular}

The current stopping patterns are skip-stop trains or all-stop trains on a 20-minute cycle (i.e., the timetable is repeated three times hourly). The cyclic structure is justified by the high frequency trains serving about 360,000 passengers daily and the operators desiring an easyto-memorise timetable (e.g., a certain train runs at 02, 22, 42). Accordingly, the cyclic structure is maintained also when solving the skip-stop optimisation and scheduled-based assignment model for this case-study. The current stopping patterns were designed individually for each railway line rather than for the corridor, and overtaking was not allowed because operationally it would translate into large dwell times for slow trains relatively to the fairly short distances in the joined sections of the corridors. Moreover, there are very few physical option for overtaking altogether, and hence overtaking is represented in this casestudy as previously described.

The implementation of the proposed bi-level optimisation problem considered the specificity of the problem, since the solutions should be feasible from the perspective of the operator and the infrastructure in order to have value and consequently potential for adoption. Train conflicts in the corridors were thus addressed by limiting the number of skipped stops between parallel railway lines, and the upper limit was selected on the basis of the number and the frequency of railway lines operated in the corridor in order to avoid conflicts for the 
optimised stopping patterns. The Danish Transport Authority estimated that skipping a stop would result in travel time savings equal to 2-3 minutes, but their estimate considered all types of trains. Accordingly, 2 minutes were considered a fair estimate for suburban trains that typically accelerate and decelerate faster than long distance trains.

\subsection{Passenger behaviour}

The sensitivity of the passengers to the various components of the generalised cost function were retrieved by empirical studies in Denmark as reported by Nielsen \& Frederiksen (2006): table 3 reports the values in terms of value of time $(\mathrm{DKK} / \mathrm{min})$ with the exception of the transfer penalty that is expressed as DKK/transfer.

Table 3. Parameter of the utility function - Beta values

\begin{tabular}{lccccrr}
\hline Purpose $c$ & $\begin{array}{l}\text { Walk time } \\
\beta_{\text {wat, }}\end{array}$ & $\begin{array}{c}\text { Waiting time } \\
\beta_{t w t, c}\end{array}$ & $\begin{array}{c}\text { Access time } \\
\beta_{a t, c}\end{array}$ & $\begin{array}{c}\text { Zone waiting } \\
\text { time } \\
\beta_{h t, c}\end{array}$ & $\begin{array}{c}\text { Transfer } \\
\text { penalty } \\
\beta_{n t, c}\end{array}$ & $\begin{array}{c}\text { In vehicle time } \\
\beta_{\text {ivt, }}\end{array}$ \\
\hline Commute & 0.280 & 0.750 & 0.633 & 0.633 & 8.800 & 0.450 \\
Business & 1.217 & 4.500 & 4.500 & 4.500 & 64.000 & 3.783 \\
Leisure & 0.117 & 0.330 & 0.467 & 0.467 & 4.000 & 0.150 \\
\hline
\end{tabular}

Three purposes were considered for this case-study. While the description of the schedule-based assignment model was general, and potentially could include as many purposes as the richness of the data would allow when estimating the parameters, the purposes available emerged from a core data source that consist in the national travel survey and the choice functions and matrices that were calibrated for the Danish National Transport Model (Rich and Hansen, 2016). Although the travel survey has more trip purposes, the number of observations for public transport trips did not allow to estimate parameters that were significant for all purposes. For example, a very limited number of shopping trips is by public transport. Accordingly, work and non-work related purposes were considered in line with the estimations presented by Anderson et al. (2017) with the addition of the distinction between commute and business trips from the values calibrated in the Danish National Transport Model.

The skip-stop optimisation affected only trains in the suburban railway network, but the schedule-based assignment considered also the other transit modes (i.e., metro, regular buses, suburban buses, express buses, regional trains, IC trains) to guarantee the maximum flexibility for the passengers to adapt their travel behaviour and hence possibly not to choose the suburban trains altogether. 
Given the demand from the Danish National Transport Model, the schedule-based assignment model was run on the basis of the utility of each traveller for each run $k$ between a certain stop $i$ and a certain stop $j$. As the bi-level problem requires the number of throughgoing, embarking, and disembarking passengers, calculations were made from the transit assignment results in order to obtain these numbers when skipping a stop. Specifically, the share of passengers from a certain stop $i$ to the skip-stop candidate $s^{*}$ for line $l$ was obtained by dividing the demand $T_{i j l c}$ from that particular stop to the candidate stop by the total demand going to the candidate stop. Then, this value was multiplied by the number $T D_{j l c}$ of disembarking passengers from railway line $l$ on the candidate stop $s$

$$
T_{i j l c}=T D_{j l c} \frac{T_{i j c}}{\sum_{i} T_{i j c}} \quad \forall i, j=s, l, c
$$

where $T_{i j l c}$ is used as input in the calculations of the additional generalised travel cost based on the applicable trip type between stops $i$ and $j$ (equations 15-20). The calculation was repeated for each railway line $l$ and trip purpose $c$, and similar calculations (for the share of passengers from the candidate stop $s$ to stop $j$ ) were performed for computing embarking passengers.

\subsection{Skip-stop patterns}

Six iterations were necessary to solve the sequential bi-level problem until no stops could be skipped beneficially. When comparing the optimised stopping patterns with the existing, the generalised travel cost was reduced by $3.2 \%$ thanks to a reduction of in-vehicle travel time equal to $5.5 \%$ to offset increases in waiting time by $1.6 \%$ and transfers by $1.4 \%$. Although the relative benefits of the optimisation appear moderate, their actual value is considerable, especially because the cost of the actual optimisation of the timetable is negligible. Scaling the actual value for the peak-hour to an entire day and then to the yearly weekdays, the total savings are about 4.2 million passenger hours that are about 2,700 working days and 76.6 million DKK per year (about 10.3 million EUR or 12.7 million USD). Clearly, this is quite a large benefit for the society at large.

Recalling that the objective was a reduction of not only travel time for passengers, but also heterogeneity of railway operations, the SSHR was computed for each of the five corridors. Table 4 presents the changes between the existing and the optimised stopping patterns, and clearly illustrates significant reductions in heterogeneity when compared to existing operations. 
Table 4. SSHR heterogeneity values in the corridors

\begin{tabular}{lrrrrr}
\hline Corridor & 1 & 2 & 3 & 4 & 5 \\
\hline All-stop & 2.40 & 1.35 & 1.35 & 0.60 & 2.40 \\
Existing & 5.00 & 1.50 & 1.70 & 0.90 & 6.00 \\
Optimised & 3.50 & 1.43 & 1.43 & 0.60 & 2.55 \\
\% optimised vs. existing & $-30.0 \%$ & $-4.7 \%$ & $-15.9 \%$ & $-33.3 \%$ & $-57.5 \%$ \\
\hline
\end{tabular}

It should be noted that train operations are homogeneous for the all-stop scenario, which is presented as a lower bound on the SSHR in each corridor. The SSHR values in table 4 depend primarily not only on the headway between subsequent trains determined by the number of railway lines operated in the corridor, but also on the difference in the number of skipped stops between railway lines in the corridor.

Table 5 compares the average number of skipped stops between the existing and the optimised patterns. When comparing the optimised stopping patterns with the existing ones, more stops were skipped in corridors 1 and 2, less stops were skipped in corridors 4 and 5, and the same number of stops were skipped in corridor 3 . When comparing the standard deviations, a significant reduction is observed for the optimised stopping patterns with respect to the existing ones: this is a result of the limit imposed on the difference in the number of skipped stops between railway lines operated in the same corridor. Even though the number of skipped stops is the same for corridor 3 for the existing and the optimised stopping patterns, the heterogeneity of operations was reduced.

Table 5. Skipped stops statistics for every corridor

\begin{tabular}{lccccccccccc}
\hline Corridor & \multicolumn{2}{c}{1} & \multicolumn{2}{c}{2} & \multicolumn{2}{c}{3} & \multicolumn{2}{c}{4} & \multicolumn{2}{c}{5} \\
\hline Railway lines operating & 4 & \multicolumn{2}{c}{3} & \multicolumn{2}{c}{3} & \multicolumn{2}{c}{2} & \multicolumn{2}{c}{4} \\
Stopping pattern & Exist. & Opt. & Exist. & Opt. & Exist. & Opt. & Exist. & Opt. & Exist. & Opt. \\
Average & 4.25 & 4.75 & 1.00 & 1.33 & 1.33 & 1.33 & 2.50 & 1.00 & 2.50 & 2.25 \\
Standard deviation & 3.50 & 0.96 & 1.73 & 0.58 & 2.31 & 0.58 & 3.54 & 0.00 & 2.89 & 0.50 \\
\hline
\end{tabular}

Figure 6 shows that the patterns are different, since in the existing ones trains were either operated as all-stop or express trains, while in the optimised ones trains were operated all as skip-stop trains. Figure 7 represents a sketch of the time-space diagram for the existing and optimised stopping patterns for the same corridor. Optimising the stopping patterns yields a wider train spread. Consequently, minor delays are more likely to be absorbed by the buffer time rather than be propagated to subsequent trains. 


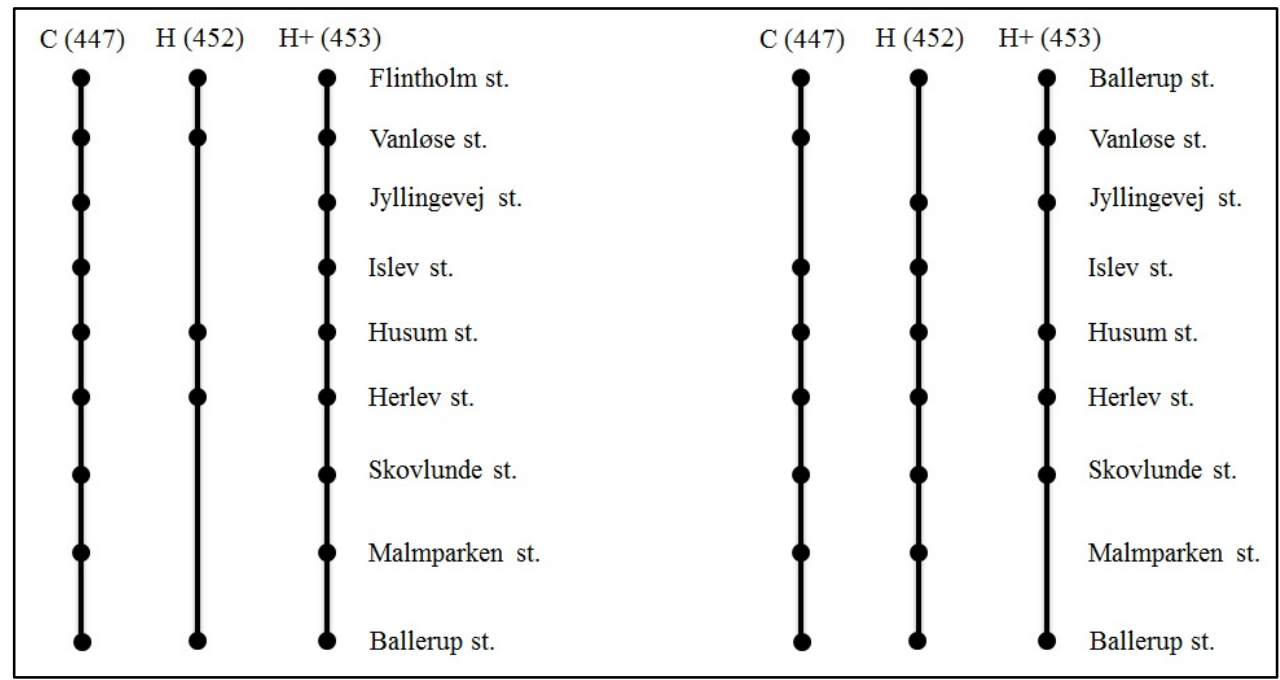

Figure 6. Line diagram for the existing (left) and optimised (right) stopping patterns in corridor 3

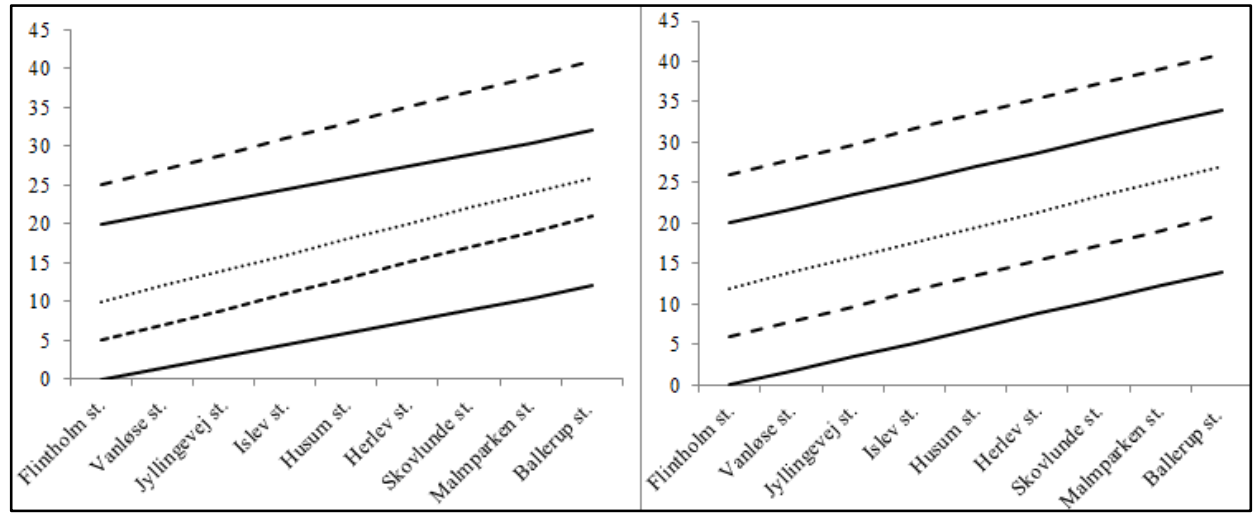

Figure 7. Time-space diagram for the existing (left) and optimised (right) stopping patterns in corridor 3

\subsection{Test of weight combinations}

A sensitivity analysis of the results was performed with respect to the weights in the objective function and the responsiveness of passenger behaviour to the changes in stopping patterns.

Table 6 presents 10 different combinations of the weights in the objective function that covers from the emphasis being mainly on the heterogeneity reduction to the emphasis being mainly to the travel time minimisation. Figure 8 illustrates how the 10 combinations perform in terms of average heterogeneity of the railway operations in the five corridors (on the horizontal axis) and total travel time for the passengers (on the vertical axis). Figure 8 shows that a $7 \%$ travel time reduction from combination 1 (favouring heterogeneity) to combination 10 (favouring travel time) implies an increase in average heterogeneity from 1.6 to 3.6. Moreover, figure 8 shows that the reduction in travel time for the passengers implies a marginal increase in heterogeneity for the first five combinations, while small reductions in travel time for the passengers imply relatively large increases in heterogeneity for the last combinations. 
Table 6. Weight combinations for sensitivity analysis

\begin{tabular}{ccc}
\hline Combination & $\omega_{1}$ (travel time) & $\omega_{2}$ (heterogeneity) \\
\hline 1 & 0.0 & 1.0 \\
2 & 0.1 & 0.9 \\
3 & 0.2 & 0.8 \\
4 & 0.3 & 0.7 \\
5 & 0.4 & 0.6 \\
6 & 0.5 & 0.5 \\
7 & 0.6 & 0.4 \\
8 & 0.7 & 0.3 \\
9 & 0.8 & 0.2 \\
10 & 0.9 & 0.1 \\
\hline
\end{tabular}

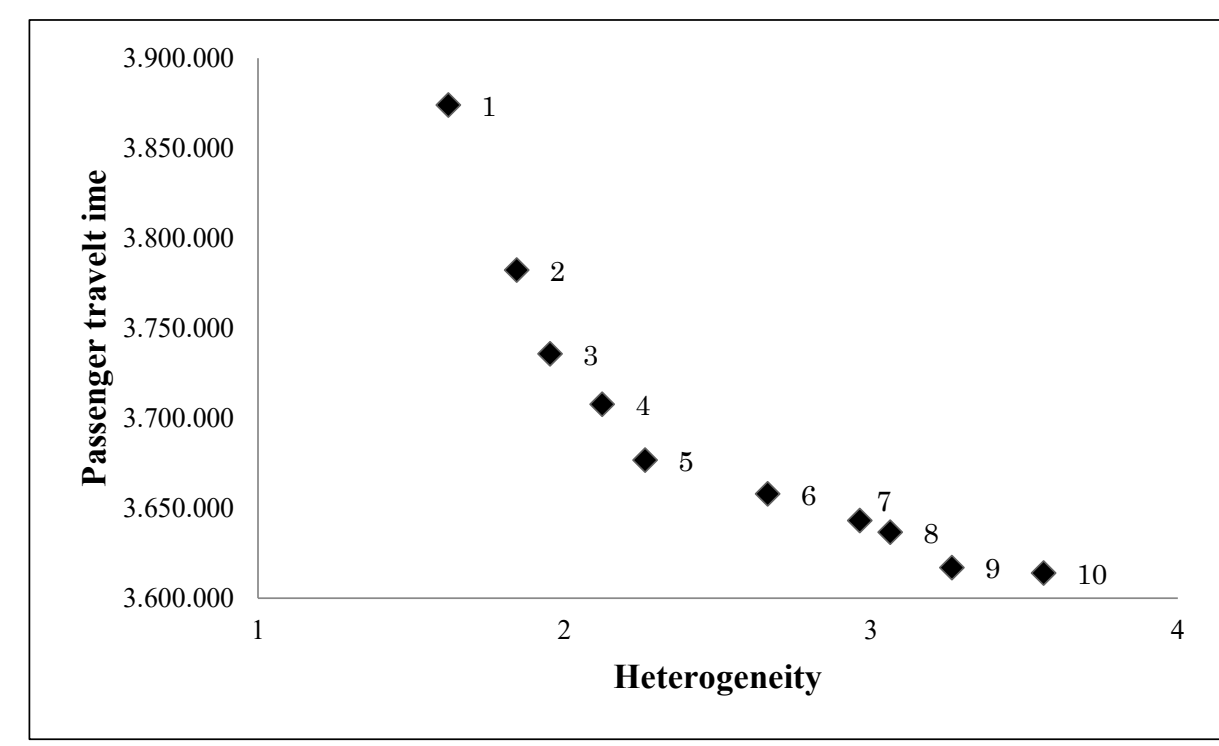

Figure 8. Pareto frontier of the sensitivity analysis to the weight combinations

\subsection{Analyses of fixed versus responsive demand}

Given that existing models do not consider responsive passenger behaviour to the changes in stopping patterns, an analysis was performed to evaluate the case where the passengers would not adapt to the changes in the timetables. Table 7 shows the variations in generalised travel cost as well as in-vehicle travel time, waiting time and number of transfers for the existing and the optimised stopping patterns with both fixed and responsive demand.

The results shows that both the total in-vehicle time and the generalised cost improve more when the optimisation considers responsive rather than fixed demand following the skip-stop optimisation. Notably, the optimisation increased the number of transfers and the waiting time as a result of skipping some stops. The benefit results from the majority of the travellers having lower in-vehicle travel time. This result is related to the specific existing 
timetable that appears to have too many stops with a wide variation in the passenger volumes across stations. In fact, the suburban trains vary from the 162 daily boarding passengers at Kildedal station to the 41,998 at Nørreport station. Moreover, the results indicate that some of the stations are served by too many lines as the overall passenger travel time and generalised cost can be improved by optimising the schedule.

Table 7. Results with fixed and responsive demand

\begin{tabular}{lrrrr}
\hline & $\begin{array}{r}\text { Transfers } \\
\text { (number) }\end{array}$ & $\begin{array}{r}\text { In-vehicle time } \\
(\mathrm{min})\end{array}$ & $\begin{array}{r}\text { Waiting time } \\
(\mathrm{min})\end{array}$ & $\begin{array}{r}\text { Generalised } \\
\text { cost (DKK) }\end{array}$ \\
\hline Existing patterns & 81,823 & $3,875,805$ & 193,393 & $2,834,874$ \\
\hline Optimised patterns, fixed demand & 82,599 & $3,725,321$ & 196,341 & $2,770,986$ \\
\% optimised vs existing, fixed demand & 0.9 & -3.9 & 1.5 & -2.3 \\
\hline Optimised patterns, responsive demand & 82,952 & $3,663,411$ & 196,487 & $2,744,188$ \\
\% optimised vs existing, responsive demand & 1.4 & -5.5 & 1.6 & -3.2 \\
\hline
\end{tabular}

\subsection{Results segmented on trip purposes}

Given the availability of data for the three trip purposes, table 8 shows the results distributed on these purposes. It should be noted that the calculation used 9 iterations and, given that the demand model implements a stochastic simulation of departure time, the solution is not exactly the same as the one presented in table 3 (also because of the extra iterations for convergence)

The table shows that all trip purposes benefit from the optimisation, although to a different extent with business trips benefitting the most and leisure trips the least. This can be intuitively explained with the fact that business trips have the highest value of time and leisure trips have the lowest, and the objective function minimises the generalised travel cost. The table also shows that all purposes are saving time and generalised travel cost. This can be intuitively explained with the fact that stations have fairly similar purpose splits, namely a station serving a smaller population will have in proportion fewer commuters, business travellers and leisure travellers in the same proportion than a station serving a larger population. 
Table 8. Results distributed on trip purposes with responsive demand

\begin{tabular}{lrrrr}
\hline & $\begin{array}{c}\text { Transfers } \\
\text { (number) }\end{array}$ & $\begin{array}{c}\text { In-vehicle } \\
\text { time (min) }\end{array}$ & $\begin{array}{c}\text { Waiting time } \\
\text { (min) }\end{array}$ & $\begin{array}{r}\text { Generalised } \\
\text { cost (DKK) }\end{array}$ \\
\hline Existing patterns, Commuter & 68,385 & $3,221,883$ & 162,057 & $2,173,181$ \\
Existing patterns, Business & 2,153 & 95,679 & 4,735 & 65,557 \\
Existing patterns, Leisure & 11,080 & 553,704 & 25,994 & 366,167 \\
\hline Total, existing & 81,619 & $3,871,265$ & 192,787 & $2,604,905$ \\
\hline Optimised, Commuter & 69,836 & $3,014,186$ & 168,951 & $2,097,652$ \\
Optimised, Business & 2,195 & 89,402 & 4,941 & 63,254 \\
Optimised, Leisure & 11,262 & 534,056 & 25,168 & 358,309 \\
\hline Total, optimised & 83,293 & $3,637,644$ & 199,060 & $2,519,215$ \\
\hline \% optimised vs existing, Commuter & 2.1 & -6.4 & 4.3 & -3.5 \\
\% optimised vs existing, Business & 1.9 & -6.6 & 4.4 & -3.5 \\
$\%$ optimised vs existing, Leisure & 1.6 & -3.5 & -3.2 & -2.1 \\
\hline Total, \% optimised vs existing & 2.1 & -6.0 & 3,3 & -3.3 \\
\hline
\end{tabular}

\section{Conclusions}

This study formulated a bi-level optimisation problem that finds optimal stopping patterns while considering responsive passenger behaviour after stops are skipped, and proposed a heuristic solution algorithm that was then applied to a large-scale suburban network with 16.8 million possible stopping configurations. The implementation focused on the suburban railway network of the Greater Copenhagen Area, which considered passengers route changes not only to re-route on the railway network, but also to other available transit modes. Moreover, the case-study presented a number of constraints to timetabling that were also accounted for in the successful implementation of the method.

Results from the optimisation yielded a significant reduction in passengers' in vehicle travel time, generalised costs and train operation heterogeneity. In fact, the spread of trains in the corridors increased and accordingly the risk of minor delays to be propagated onto subsequent trains decreased. In a nutshell, railway passengers could move faster between stop $i$ and stop $j$ while incurring in a lower risk of delays. Considering the adapted route choices to the stopping patterns was also beneficial, in particular because the in-vehicle times were significantly reduced with respect to the case where the demand would be fixed. Overall, the reduction of passengers' in-vehicle time reached 5.5\% and the yearly consumer surplus amounted at 76.7 million DKK (about 10.3 million EUR or 12.7 million USD).

Future research could look at further improvement in the heuristic optimisation of the upper level problem, as there could be instances in which the heuristic does not reach the optimal solution and more advanced metaheuristics may be developed further. Another avenue for future development of the algorithm would be to generate smart initial solutions, given that the bi-level problem is affected by the initial solution for what concerns the 
network and the demand. Last, further research could look at timetable adaptation (similar to Parbo et al., 2014) being combined with the skip-stop optimisation since both aim at improving transit operations from the passengers' perspective. Looking at the optimisation potential of combining these two models sequentially could be interesting in light of the fact that the 5.5\% reduction in in-vehicle time from the skip-stop optimisation could be matched to the $5.1 \%$ reduction in transfer time from the timetable adaptation in the same network. Potentially, there is a larger total benefit for the passenger when combining the two models.

\section{Acknowledgements}

We gratefully acknowledge the financial support of the Danish Council for Strategic Research for the project "RobustRailS" that this study is part of. We also thank three anonymous reviewers for the insightful comments that helped improving a previous version of the paper.

\section{References}

Anderson, M.K., Nielsen, O.A., Prato, C.G., 2017. Multimodal route choice models of public transport passengers in the Greater Copenhagen Area. EURO Journal in Transportation and Logistics, 6, 221-245.

Borndörfer, R., Grötschel, M., Pfetsch, M.E., 2007. A column-generation approach to line planning in public transport. Transportation Science, 41, 123-132.

Bull, S.H., Lusby, R.M., Larsen, J., 2015. An optimization based method for line planning to minimize travel time. Proceedings of the 13th Conference on Advanced Systems in Public Transport, Delft, The Netherlands.

Ceder, A., 2015. Public Transit Planning and Operation: Modeling, Practice and Behavior. CRC Press, Boca Raton, FL.

Chen, X., Hellinga, B., Chang, C., Fu, L., 2015. Optimization of headways with stop skipping control: a case study of bus rapid transit system. Journal of Advanced Transportation, 49, 385-401.

Constantin, I., Florian, M., 1995. Optimizing frequencies in a transit network: a nonlinear bilevel programming approach. International Transactions in Operational Research, 2 , 149-164.

Feng, S., Wen-tao, Z., Ying, Y., Dian-hai, W., 2013. Optimal skip-stop schedule under mixed traffic conditions for minimizing travel time of passengers. Discrete Dynamics in Nature and Society, 2013, article 930562.

Freyss, M., Giesen, R., Muñoz, J.C., 2013. Continuous approximation for skip-stop operation 
in rail transit. Transportation Research Part C: Emerging Technologies, 36, 419-433.

Fu, H., Nie, L., Meng, L., Sperry, B.R., He, Z., 2016. A hierarchical line planning approach for a large-scale high speed rail network: The China case. Transportation Research Part A: Policy and Practice, 75, 61-83.

Gentile, G., Noekel, K., 2016. Modelling public transport passenger flows in the era of Intelligent Transport Systems. Springer Tracts on Transportation and Traffic, Springer, Switzerland.

Jamili, A., Ghannadpour, S.F., Ghorshinezhad, M., 2014. The optimization of train timetable stop-skipping patterns in urban railway operations. In: Brebbia, C.A., Tomii, N., Tzieropoulos, P., Mera, J.M. (Eds), Computers in Railways XIV: Railway Engineering Design and Optimization, Volume 135, WIT Press, Southampton, UK, pp. 603-611.

Jong, J.C., Suen, C.S.J., Chang, S.K.J., 2012. Decision support system to optimize railway stopping patterns. Transportation Research Record, 2289, 24-33.

Katori, T., Kabumoto, H., Izumi, T., 2014. Shortening average trip times by adjusting stopping and overtaking train stations. In: Brebbia, C.A., Tomii, N., Tzieropoulos, P., Mera, J.M. (Eds), Computers in Railways XIV: Railway Engineering Design and Optimization, Volume 135, WIT Press, Southampton, UK, pp. 577-586.

Kikuchi, S., Vuchic, V.R., 1982. Transit vehicle stopping regimes and spacings. Transportation Science, 16, 311-331.

Lee, Y.J., Shariat, S., Choi, K., 2014. Optimizing skip-stop rail transit stopping strategy using a genetic algorithm. Journal of Public Transportation, 17, 135-164.

Leiva, C., Muñoz, J.C., Giesen, R., Larrain, H., 2010. Design of limited-stop services for an urban bus corridor with capacity constraint. Transportation Research Part B: Methodological, 44, 1186-1201.

Lin, D.Y., Ku, Y.H., 2014. Using genetic algorithms to optimize stopping patterns for passenger rail transportation. Computer-Aided Civil and Infrastructure Engineering, 29, 264-278.

Mesa, J.A., Ortega, F.A., Pozo, M.A., 2009. Effective allocation of fleet frequencies by reducing intermediate stops and short turning in transit systems. In: Ahuja, R., Möhring, R.H., Zaroliagis, C.D. (Eds), Robust and Online Large-Scale Optimization, Springer, Berlin, Germany, pp. 293-309.

Nielsen, O.A., 1998. Two new methods for estimating trip matrices from traffic counts. In: Ortúzar, J. de D., Hensher, D., Jara-Díaz, S. (Eds), Travel Behaviour Research: Updating the State of Play, Elsevier, Oxford, UK, pp. 221-250. 
Nielsen, O.A., 2000. A stochastic transit assignment model considering differences in passengers' utility functions. Transportation Research Part B: Methodological, 34, 377402.

Nielsen, O.A., 2004. A large scale stochastic multi-class schedule-based transit model with random coefficients. In: Wilson, N.H., Nuzzolo, A. (Eds), Operations Research/Computer Science Interfaces Series, Vol. 28, Springer Science, New York, NY, pp. 53-77.

Nielsen, O.A., Frederiksen, R.D., 2006. Optimisation of timetable-based, stochastic transit assignment models based on MSA. Annals of Operations Research, 144, 263-285.

Nielsen, B.F., Frølich, L., Nielsen, O.A., Filges, D., 2014. Estimating passenger numbers in trains using existing weighing capabilities. Transportmetrica A: Transport Science, 10, 502-517.

Parbo, J., Nielsen, O.A., Prato, C.G., 2014. User perspectives in public transport timetable optimisation. Transportation Research Part C: Emerging Technologies, 48, 269-284.

Raveau, S., Guo, Z., Muñoz, J.C., Wilson, N.H., 2014. A behavioural comparison of route choice on metro networks: Time, transfers, crowding, topology and socio-demographics. Transportation Research Part A: Policy and Practice, 66, 185-195.

Rezanova, N.J., 2015. Line planning optimization at DSB. Proceedings of the 13th Conference on Advanced Systems in Public Transport, Delft, The Netherlands

Rich, J., Hansen, C.O., 2016. The Danish national passenger model - Model specification and results. European Journal of Transport and Infrastructure Research, 16, 573-599.

Schöbel, A., Scholl, S., 2006. Line planning with minimal traveling time. In: Kroon, L.G., Möhring, R.H. (Eds), 5th Workshop on Algorithmic Methods and Models for Optimization of Railways, Schloss Dagstuhl, Dagstuhl, Germany, doi:10.4230/OASIcs.ATMOS.2005.660.

Sogin, S.L., Caughron, B.M., Chadwick, S.G., 2012. Optimizing skip stop service in passenger rail transportation. Proceedings of the 2012 Joint Rail Conference, Philadelphia, PA.

Suh, W., Chon, K.S., Rhee, S.M., 2002. Effect of skip-stop policy on a Korean subway system. Transportation Research Record, 1793, 33-39.

Sun, L., Jin, J.G., Lee, D.H., Axhausen, K.W., Erath, A., 2014. Demand-driven timetable design for metro services. Transportation Research Part C: Emerging Technologies, 46, 284-299.

Tetreault, P.R., El-Geneidy, A.M., 2010. Estimating bus run times for new limited-stop service using archived AVL and APC data. Transportation Research Part A: Policy and 
Practice, 44, 390-402.

Vromans, M.J., Dekker, R., Kroon, L.G., 2006. Reliability and heterogeneity of railway services. European Journal of Operational Research, 172, 647-665.

Wang, J.Y., Lin, C.M., 2010. Mass transit route network design using genetic algorithm. Journal of the Chinese Institute of Engineers, 33, 301-315. 